\title{
Approximating Heart Rate Variability
}

\author{
Jen-Liang Cheng, Ph.D. \\ Dept. of Medical Informatics \\ Tzu-Chi University \\ Hua-Lien City, Taiwan. \\ tomcheng@mail.tcu.edu.tw
}

\author{
Jin-Ren Jeng, M.D. \\ Division of Cardiology \\ Tzu-Chi General Hospital \\ Hua-Lien City, Taiwan. \\ jrjeng@tzuchi.com.tw
}

\author{
Zhu-Xuan Lin \\ Dept. of Medical Informatics \\ Tzu-Chi University \\ Hua-Lien City, Taiwan. \\ zxlin67@yahoo.com.tw
}

\author{
Jiunn-Horng Lee \\ Dept. of Medical Informatics \\ Tzu-Chi University \\ Hua-Lien City, Taiwan. \\ 96325123@stmail.tcu.edu.tw
}

\begin{abstract}
Heart rate variability (HRV) measurement in the field has not been widely studied due to the presence of substantial noises in certain circumstances even after signal processing. To overcome such a difficulty, a method, called VACA (Vote-AndChain Algorithm) is proposed to obtain an approximate HRV measurement. With VACA, the contaminated ECGs can be patched to obtain HRV metric, such as SDNN, even when the arrival rate of noises has reached the same level of heart rate. The performance of this algorithm is evaluated with 27,000 contaminated ECGs which are synthesized by real ECGs in the Physio-Net and noises of Poisson process. The best parameters for VACA are explored so that it can reach an accuracy of $(100 \pm 20) \%$ for $97 \%$ of the 27000 contaminated ECG data. The experiment results show that VACA is an robust method for HRV measurement in applications that long-term multi-lead ECG is not feasible.
\end{abstract}

Keywords-Heart Rate Variability; Robustness; ECG; Approximation

\section{INTRODUCTION}

HRV(Heart Rate Variability) represents one of the most promising marker for the relationship between the autonomic nervous system and cardiovascular mortality. The apparently easy derivation of this measure has popularized its use. Many commercial devices now provide an automated measurement of HRV, the cardiologist has been provided with a seemingly simple tool for both research and clinical studies[1]. Because of its demanded accuracy, current HRV devices adopts signal processing methods for noise elimination and inter-beat interval acquisition.

The demand of HRV accuracy needs a certain amount of computing power, data communication, and/or data storage. To avoid the generation of noises, short term HRV measurement requires that the tested objective being in a dormant posture during the recording period of electrocardiogram(ECG). For a long-term HRV, twenty-four hour ECG must be recorded with a multiple-lead Holter monitor. To measure HRV, the pulse train of R-peak for a specified period must be detected or calculated to proceed the following HRV analysis. The above recorded ECG then must be processed by several signal processing routines such as filtering and peak detection to obtain the pulse train of heartbeat for following HRV analysis.
Because HRV is capable of revealing some associated chronicle disorders, it is desirable to leverage it in application of preventive and elderly medicine, such as homecare or telecare metrics. In these applications, the apparatus to be worn should be the one with tiny size, low power requirement, and robustness. The demand of a series of signal processing routines make the HRV measurement difficult to be implemented as a wearable device.

Rather than an HRV device with high accuracy, we urge that an robust HRV device should also be developed for more other applications. With the robustness, the requirement of computing, communication, and storage may be reduced. For example, a peak detecting circuit rather than a sampling analog/digital converter as well as noise eliminating routines can be used for signal detection and processing. On measuring heart beats of a moving object with above simple front-end circuitry, it is unlikely that noises can be filtered by conventional procedure. Thus a mixture of heart beats and noises are obtained. Because the noises can not be differentiated from R-peaks, the HRV measurement may be shifted.

To resolve the difficulty mentioned, a method to pick up heartbeats and then to approximate original HRV from the mixture is desirable. As can be seen from the simulation results, the very-long (in weeks) or in-the-field HRV measurement can be feasible with such a method that implemented in a wearable device.

\section{The Vote-And-Chain Algorithm}

The proposed Vote-And-Chain Algorithm(VACA) is based the heart rate approximation algorithm[2]. In that algorithm, the events of heartbeats or noise are collected and processed for every, say, 4 seconds. Following paragraphs briefs how it works.

During the period that events (R-peaks and noises) are collected all inter-event intervals between any two events are calculated. In the end of the collecting period, a voting process is held. There are tens of ballot boxes, say 64, that inter-event intervals vote. Each box is labeled with a range of possible RR interval's length so that all boxes consists of the possible RR interval of a human. Then each of the intervals are voted

Granted by NSC, Taiwan. Gant\#:NSC96-2219-E-320-001

Permission to make digital or hard copies of all or part of this work for personal or classroom use is granted without fee provided that copies are not made or distributed for profit or commercial advantage and that copies bear this notice and the full citation on the first page. To copy otherwise, to republish, to post on servers or to redistribute to lists, requires prior specific permission and/or a fee. PERVASIVEHEALTH 2008, 30 Jan - 1 Feb. Tampere, Finland

Copyright $\odot 2008$ ICST 978-963-9799-15-8

DOI 10.4108/ICST.PERVASIVEHEALTH2008.2552 
to the associated box according to their length. For any healthy human, the length of consecutive RR intervals can vary only slightly. Thus, within a short period, the intervals that formed by the two consecutive R-peaks are more likely to be clustered together than intervals consists of a noise. The clustered intervals are then speculated as the real R-peaks.

Fig. 1 shows an example for that $H R$ approximation algorithm. As can be seen from Fig. 1, events $E_{1}-E_{6}$ occurred in the time window $W_{1}$ (with length of $T_{\mathrm{w}}$ ).

Without the loss of generality, the starting of the $W_{1}$ is on the time that $E_{1}$ occurred. Of those events, $E_{2}$ and $E_{6}$ are noises. Then all intervals between any two events are calculated. That is,

$$
I_{\mathrm{i}, \mathrm{j}}=t\left(E_{\mathrm{j}}\right)-t\left(E_{\mathrm{i}}\right), \mathrm{j}>\mathrm{i}
$$

are evaluated, where $t\left(E_{\mathrm{i}}\right)$ is the time that $E_{\mathrm{i}}$ occurred.

After precluding the intervals that cannot be a normal RRinterval, each inter-event intervals is voted to the associated ballot box according to their length, as shown in Fig. 2. In the figure, each ballot box is labeled with the range to which its voters(the inter-event intervals) belong. For simplicity, the range is an exponential number, say $2^{B} \mathrm{~ms}$, where $B$ is the exponent of the range of a ballot box. Note that intervals that outrange the reasonable R-R interval are precluded for voting. For the example above, $\mathrm{I}_{1,2}, \mathrm{I}_{2,3}, \mathrm{I}_{5,6}$, and $\mathrm{I}_{6,7}$ are precluded and not voted to ballot boxes.

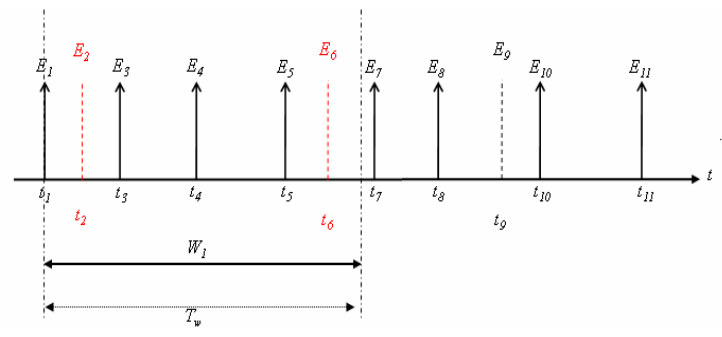

Fig. 1. An example for HR Approximation algorithm.

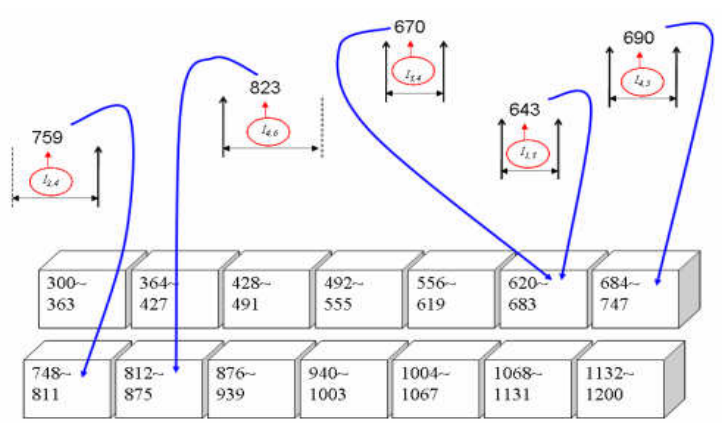

Fig. 2. How Interevent intervals vote to the different ballots.

Finally, every two neighboring boxes' votes are compared. The collaborated votes that wins the voting are then speculated to be the RR-intervals. In Fig. 2, the speculated RR intervals are the three inter-event intervals that voted to ballots with labels of 620-683 and 684-747. And events that formed these intervals are speculated as R-peak events.

Two problem arise when the pulse train obtained by above algorithm is to be used for measuring HRV. First, if more than one interval groups have the highest number of votes, which group should be chosen? Second, since the contiguous windows are independent, how to chain them to form a series of R-peaks. The solution to these two problems is the overlapped window scheme.

With the overlapped window scheme, a new window begins its event collecting process before the previous window finishes the voting, as shown in Fig. 3. The overlapped time zone, depicted as $T_{\text {overlap }}$, is the period that time windows $W_{1}$ and $W_{2}$ may have the common events, which are $E_{5}$ and $E_{6}$ in this example. If two contiguous windows agree on R-peaks that reside in the overlapped zone, then all R-peaks of the two windows are chained to form a longer series.

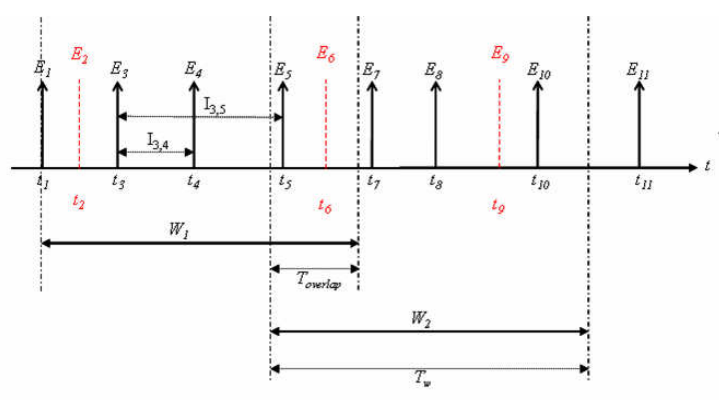

Fig. 3. An example of overlapped window scheme.

The chaining process not only forms a longer series, it helps in choosing the most probable R-peak group when an equivocal condition occurs. The reason is that the real R-peaks may form a longer series than noises do.

Because noises do exist, there are chances that two windows do not agree on R-peaks in overlapped zone. In that case, the previous R-peak series are, unfortunately, fractured. A higher noise rate may generate more shorter and fractured series as can be seen from the simulation results.

\section{SimUlation ResUlts}

\section{A. Simulation Data and Methods}

The database employed to evaluate VACA is the normal sinus rhythm RR interval database of PhysioNet[4], which consists of ECGs of 54 objectives without cardiovascular diseases. Of the 54 objectives, 24 are females, 30 are males. Of their ages, the mean is 61.36 , the standard deviation is 11.63 , the maximal is 76 , and the smallest is 28.5 .

Of each ECG of 24 hours, first thirty minutes are extracted and contaminated by the noise generator for later simulation.

The noises generator is in fact a Poisson process for the reason of its random and memory-less characteristics. The performance of VACA is evaluated for different noise arrival rates (or noise rates) ranging from 0.01 to 1.00 with step of 0.01 . Because pseudo random number is employed to generate a noise event, the measurement of the same performance metric on a noise rate is iterated five times so that VACA's 
performance can be evaluated fair. Thus 500 iterations required for each 30-min ECG.

Since a contaminated R-peak series could be fractured by $\mathrm{VACA}$, a patchery process is required to piece together those fractured series so that approximate HRV metrics can be analyzed. The HRV metric used to evaluate the accuracy of VACA is SDNN for its popularity and simplicity. SDNN, stands for Standard Deviation of NN interval, is long for HRV measures and is scale-dependent.

VACA's performance is evaluated as below: The original SDNN for the first 30-min ECG is evaluated first. Then the ECG is contaminated by Poisson process with a specified noise rate. Piece-wise R-peak series can be obtained after the contaminated series is processed by VACA. After piecing together those fractured R-peak series, the SDNN of the patched RR intervals, called estimated $\mathrm{SDNN}(\mathrm{E}-\mathrm{SDNN})$, is calculated and then divided by the original SDNN, the ratio is called E-SDNN accuracy. As mentioned, this procedure will be executed five times to mimic the possible circumstances.

Fig. 4 shows an example of E-SDNN accuracy under different noise rates for first five minutes ECG of objective NSR034. When an E-SDNN accuracy is obtained a crisscross is marked for the specific noise rate. As can be seen from the figure, there are 5 crisscross marks for each noise rate. Thus, 500 crisscross marks can be found in this figure.

In Fig. 4 two dashed lines have enclosed the accuracy level between $80 \%$ and $120 \%$. The crisscross mark enclosed between dashed lines is said to be "acceptable". And if the averaged ESDNN accuracy for a noise rate is acceptable, then we can say that VACA is effective in obtaining HRV for a contaminated ECG.

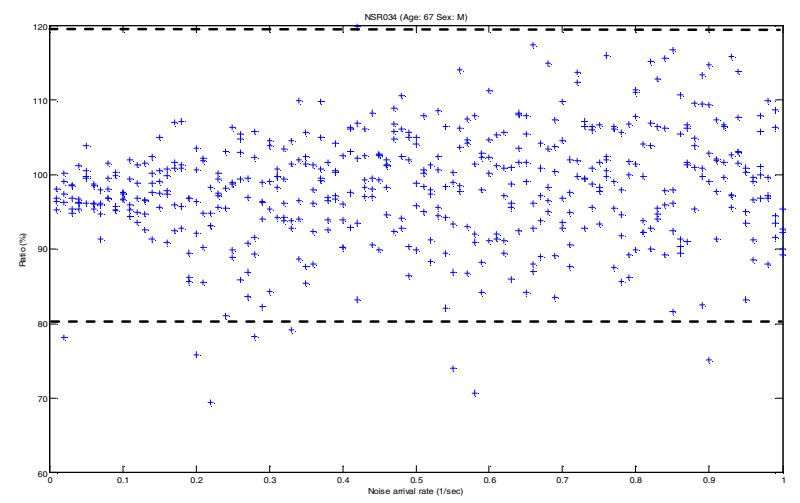

Fig. 4. An example of E-SDNN accuracy after VACA( ECG NSR034)

\section{B. Optimal Parametes for VACA}

VACA's performance is determined by following parameters: time window length $T_{\mathrm{w}}$, the length of overlapped zone $T_{\text {overlap, }}$ and the range of a ballot box $2^{B}$, all in millisecond(ms). For each parameter combination, ECGs of 54 objectives for 100 noise rates are simulated to test the E-SDNN accuracy. A parameter combination with higher number of accepted E-SDNN accuracy for all noise rates is more desirable.
Our simulation results show that best results can be obtained with following parameter combinations[3]:

$$
\begin{aligned}
& -T_{w}=4000, T_{\text {overlap }}=1500, B=6 \\
& -T_{w}=4000, T_{\text {overlap }}=2000, B=6 \\
& -T_{w}=5000, T_{\text {overlap }}=2500, B=6
\end{aligned}
$$

Fig. 5 shows the summarized result for these three parameter combinations, which shows that the number of ECGs with its contaminated counterpart's E-SDNN accuracy is accepted. Among which, the combination of 4000-1500-6 has the highest acceptance rate (97\%) for the 27,000 contaminated ECGs.

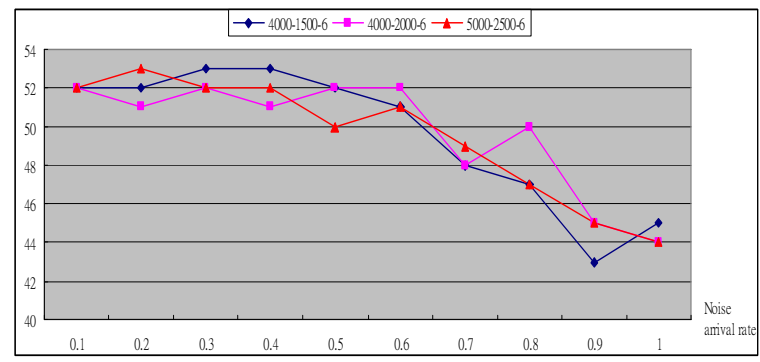

Fig. 5. Accepted number of ECGs with different parametric combinations under various noise rates. [3]

\section{The E-SDNN Accuracy with VACA s}

The averaged E-SDNN accuracy are presented in Fig. 6. In the figure, all three parameter combinations can achieve quite good accuracy even when the noise rate has reached to 1.00 which approximate the sedentary heart rate of some healthy human.

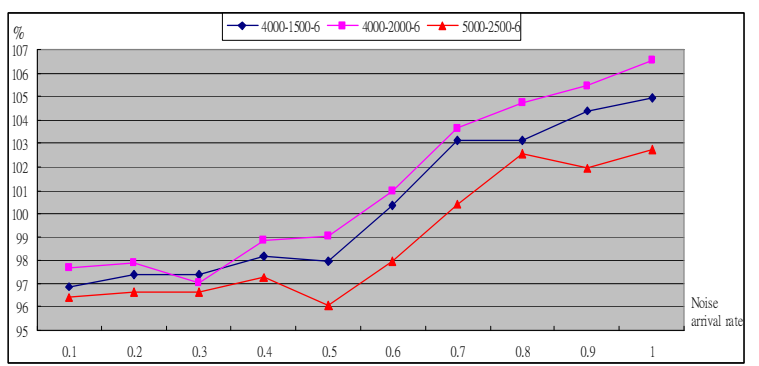

Fig. 6. Average accuracy of E-SDNN under various noise rates and parameter combinations.

In summary, the combination of 4000-1500-6 can achieve the best E-SDNN accuracy when noise rate is less than 0.5 . When noise rate is more than 0.5 , better results can be obtained with the combination of 4000-2000-6. The combination of 5000-2500-6 performs almost as well as the other two combinations with better robust characteristic for even higher noise rate.

\section{The Exception}

As Fig. 5 has shown, at most 53 ECGs are accepted for any parameter combinations. The exceptional ECG has been 
identified with its tachogram of RR intervals shown in Fig. 7.

In this case, the original SDNN is extraordinarily large, so that more than one majority groups are formed. Thus VACA may fail to choose the group with highest votes during the voting phase. The problem may be alleviated by clustering 3 rather than 2 neighboring ballot boxes for majority. This alternative, however, affects the E-SDNN's accuracy associated with other ECGs. That means that VACA has faced a dilemma in tuning parameters between exceptional ECGs and ordinary ECGs

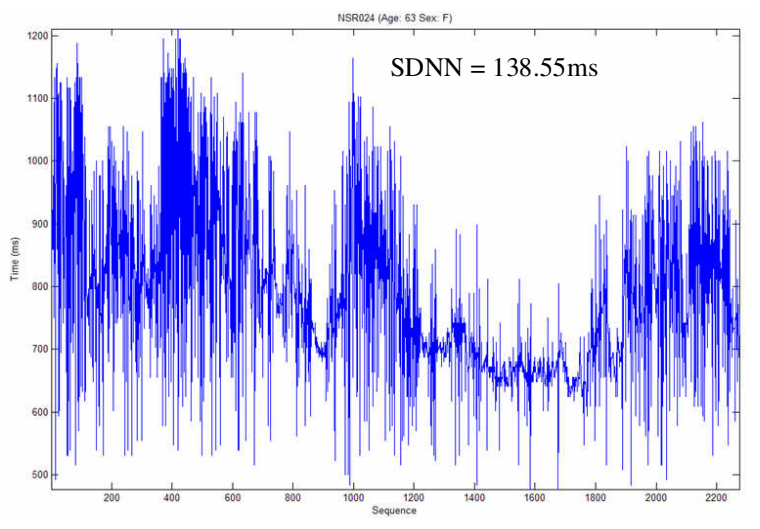

Fig.7. Tachogram of RR series of NSR024.

\section{CONCLUSIONS}

Since the significance of HRV measurement in clinic has been proved and well documented for the last two decades. The applications of HRV in homecare or telecare is foreseeable. For these applications, an all-day-long or an in-the-field HRV monitor may be required

With VACA, alternative front end, e.g. a peak detector or contactless methods, is possible. And its performance and robustness have been shown with the data from PhysioNet.

[1] L. S. Dreifus, J. B. Agarwal, E. H. Botvinick, etc., "Heart rate variability for risk stratification of life-threatening arrhythmias," J Am Coll Cardiol. Vol. 22, pp. 948-950, 1993.

[2] Jen-Liang Cheng, etc., "Heart Rate Measurement in the Presence of Noises", Proceedings of 1 st International Conference on Pervasive Computing Technologies for Healthcare, Innsbruck, Austria, 2006.

[3] Z. X. Lin "The Study of Two Level Qualitative Herat Rate Variability Estimation Algorithm," Master Thesis of Tzu-Chi University, August, 2007.

[4] Goldberger AL, Amaral LAN, Glass L, Hausdorff JM, Ivanov PCh, Mark RG, Mietus JE, Moody GB, Peng CK, Stanley HE. PhysioBank, PhysioToolkit, and PhysioNet: Components of a New Research Resource for Complex Physiologic Signals. Circulation 101(23):e215e220 [Circulation Electronic http://circ.ahajournals.org/cgi/content/full/101/23/e215]; 2000 (June 13). 